\title{
Preface to the Special Issue "Recent developments in non-linear and global optimization"
}

\author{
Yaroslav D. Sergeyev ${ }^{1,2}$
}

Received: 18 December 2015 / Accepted: 22 December 2015 / Published online: 13 January 2016 (C) Springer-Verlag Berlin Heidelberg 2016

Non-linear and global optimization is a rapidly growing field of optimization dealing with theoretical, computational, and applied aspects of problems arising when people face the necessity to find optimal in some sense solutions. The importance of non-linear models continuously increases in our more and more complex world. As a consequence of this growing complexity very often objective functions are not only non-linear but also multiextremal, i.e., they can have several local optima and it is required to find the global one since the global solution can give a significant improvement with respect to the local ones.

The core of this special issue consists of papers presented at the International Conference "Numerical Computations: Theory and Algorithms" held in Falerna, Italy in June 2013. The goal of the Conference was to create a multidisciplinary round table for an open discussion on numerical computations by using traditional and emerging computational paradigms. Numerical algorithms for solving non-linear and global optimization problems were among the main topics of the conference.

Below we give a brief presentation of papers included in this special issue.

- The paper [1] by Lucidi, Maurici, Paulon, Rinaldi, and Roma proposes a derivativefree approach for a simulation-based optimization problem in healthcare. Hospitals have been challenged in recent years to deliver high quality care with limited resources. Given the pressure to contain costs, developing procedures for optimal resource allocation becomes more and more critical in this context. The authors develop a simulation-based optimization approach for the resource planning of a specific hospital ward and report results or a real-world problem coming from

$凶$ Yaroslav D. Sergeyev yaro@dimes.unical.it

1 University of Calabria, Via Pietro Bucci 42C, 87036 Rende, CS, Italy

2 Lobachevsky State University, Gagarin Av. 23, 603950 Nizhni Novgorod, Russia 
the obstetrics ward of an Italian hospital showing both the effectiveness and the efficiency of the proposed approach.

- Paulavicius and Zilinskas discuss in their paper [2] advantages of simplicial partitioning for Lipschitz global optimization problems with linear constraints showing that simplicial partitioning may tackle linear constraints in a very subtle way. An extensive experimental investigation reveals advantages of this approach comparing with different constraint-handling methods, proposed for use with DIRECT global optimization algorithm. The authors present also very competitive results with respect to a derivative-free particle swarm algorithm.

- The paper [3] by Shary and Sharaya considers the problem of recognizing solvability (nonemptiness of the solution set) for interval systems of linear algebraic equations. The authors introduce a quantitative measure of the membership of a point in the solution set reformulating their problem as a global maximization of a functional. They show that the specific value of the maximum and its argument provide them with an important quantitative information of the solvability supply or its deficiency.

- Pham Dinh, Le Thi, Pham, and Niu investigate in their article [4] modeling discrete portfolio optimization problems under concave transaction costs as DC programs. DC reformulations are established by using penalty techniques in DC programming. Then a suitable global optimization Branch and Bound technique is developed and numerical simulations showing the efficiency of computed solutions compared to standard algorithms for nonconvex nonlinear integer programs are reported.

- Guzman, Faruque Hasan, and Floudas study in their paper [5] performance of convex underestimators in a branch-and-bound framework. It is well known that the efficient determination of tight lower bounds in branch-and-bound algorithms is crucial for the global optimization. The authors explore the performance of their $\alpha \mathrm{BB}$ method and a number of competing methods using a test suite of 40 multivariate, box-constrained, nonconvex functions comparing the tightness of generated underestimators and the efficiency of convergence of global optimization algorithms.

- The paper [6] written by Cerrone, Cerulli, and Gaudioso presents a genetic algorithm called by the authors OMEGA (One Multi Ethnic Genetic Approach). The main difference with respect to existing genetic methods is that, starting from an initial population, $k$ different sub-populations are produced at each iteration and they independently evolve in $k$ different environments. The resulting sub-populations are then recombined and the process is iterated. The new algorithm is compared with several approaches drawn from the literature showing a promising performance.

- Global optimization of mathematical programs with complementarity constraints and an application to clean energy deployment is studied in the paper [7] of Zhang and Sahinidis. The authors apply a game theoretic approach to model capacity investments and new technology introduction in response to carbon emission regulations. They report algorithmic advancements that were made to enhance the performance of the deterministic global optimizer BARON on this and other mathematical programs with complementarity constraints.

- The paper [8] by Zilinskas and Zhigljavsky considers an approach to non-convex multi-objective optimization problems where only the values of objective functions are required by the algorithm. The proposed approach is a generalization of the probabilistic branch-and-bound approach well applicable to complicated problems 
of single-objective global optimization. The authors discuss the concept of probabilistic branch-and-bound based multi-objective optimization algorithms and report some illustrative examples.

- Optimizing sensor cover energy via DC Programming is the subject of the article [9] by Astorino and Miglionico who consider the problem of determining the radius of a given number of sensors, covering a set of targets, with the objective of minimizing the total coverage energy consumption. To solve the resulting problem having a nonlinear objective function and non-convex constraints the authors adopt a penalty function approach which allows them to state the problem in DC form. Numerical results using a set of randomly generated test problems are presented.

- Orlov, Strekalovsky, and Batbileg consider the problem of numerical finding a Nash equilibrium in a 3-player polymatrix game in their paper [10]. The authors show that this problem is equivalent to a nonconvex optimization problem with a bilinear structure in the objective function. Local and global optimization algorithms for this optimization problem are proposed and investigated both theoretically and numerically.

- Tröltzsch in her article [11] presents a sequential quadratic programming algorithm for equality-constrained optimization without derivatives. The algorithm constructs local polynomial interpolation-based models of the objective and constraint functions and computes steps by solving QP sub-problems inside a region using the standard trust-region methodology exploiting a self-correcting property of the interpolation set geometry. The author shows how the implementation of such a method can be enhanced to outperform well-known DFO packages on smooth equalityconstrained optimization problems.

- The article [12] by Steihaug and Suleiman discusses final steps of Newton and higher order methods. The local behavior for the unconstrained optimization problem is investigated by considering problems with two unknowns and it is shown that there are no significant differences in the region where the global method turns into a local search for second and third order methods. Final steps to reach a predefined tolerance are investigated and it is shown that the region where the higher order methods terminate in one or two iterations is significantly larger than the corresponding region for Newton's method.

- Guérard and Tseveendorj in their paper [13] consider the problem of finding a global maximum of quadratic convex maximization problems over a polytope. The authors use the largest inscribed ball for an inner approximation while a minimal enclosing box is exploited for an outer approximation of the domain and apply local search methods in a combination with some cutting plane techniques.

It is with the deep regret that I have to inform readers that during the preparation of this special issue, in January 2015, one of its authors, Professor Irene A. Sharaya, succumbed to cancer. I grieve about her together with her husband and co-author Professor S.P. Shary, their three children, two grandchildren, relatives, friends, and students. Since 1995 Professor Sharaya worked at the Institute of Computational Technologies of Russian Academy of Sciences and at Novosibirsk State University in Russia. Before that she was at the Computational Center of Russian Academy of Sciences in Kras- 
noyarsk. Professor Sharaya was very well known internationally for her methods and codes in interval analysis and optimization.

I would like to conclude this Preface by expressing my deep gratitude to the Co-Editors-in-Chief of Optimization Letters Professors Pavlo Krokhmal and Oleg Prokopyev for their continuous cordial support. I thank all the authors and the anonymous reviewers who worked on this special issue for their efforts that I hope have led to an interesting and important volume of contributions to theory and practice of non-linear and global optimization.

\section{References}

1. Lucidi, S., Maurici, M., Paulon, L., Rinaldi, F., Roma, M.: A derivative-free approach for a simulationbased optimization problem in healthcare. Optim. Lett. doi:10.1007/s11590-015-0905-4

2. Paulavicius, R., Zilinskas, J.: Advantages of simplicial partitioning for Lipschitz optimization problems with linear constraints. Optim. Lett. doi:10.1007/s11590-014-0772-4

3. Shary, S.P., Sharaya, I.A.: On solvability recognition for interval linear systems of equations. Optim. Lett. doi:10.1007/s11590-015-0891-6

4. Pham Dinh, T., Le Thi, H.A., Pham, V.N., Niu, Y.-S.: DC programming approaches for discrete portfolio optimization under concave transaction costs. Opim. Lett. doi:10.1007/s11590-015-0931-2

5. Guzman, Y.A., Faruque Hasan, M.M., Floudas, C.A.: Performance of convex underestimators in a branch-and-bound framework. Optim. Lett. doi:10.1007/s11590-014-0799-6

6. Cerrone, C., Cerulli, R., Gaudioso, M.: OMEGA—one multi ethnic genetic approach. Optim. Lett. doi:10.1007/s11590-015-0852-0

7. Zhang, Y., Sahinidis, N.V.: Global optimization of mathematical programs with complementarity constraints and application to clean energy deployment. Optim. Lett. doi:10.1007/s11590-015-0880-9

8. Zilinskas, A., Zhigljavsky, A.: Branch and probability bound methods in multi-objective optimization. Optim. Lett. doi:10.1007/s11590-014-0777-z

9. Astorino, A., Miglionico, G.: Optimizing sensor cover energy via DC programming. Optim. Lett. doi:10.1007/s11590-014-0778-y

10. Orlov, A.V., Strekalovsky, A.S., Batbileg, S.: On computational search for Nash equilibrium in hexamatrix games. Optim. Lett. doi:10.1007/s11590-014-0833-8

11. Tröltzsch, A.: A sequential quadratic programming algorithm for equality-constrained optimization without derivatives. Optim. Lett. doi:10.1007/s11590-014-0830-y

12. Steihaug, T., Suleiman, S.: On the final steps of Newton and higher order methods. Optim. Lett. doi:10. 1007/s11590-015-0899-y

13. Guérard, G., Tseveendorj, I.: Inscribed ball and enclosing box methods for the convex maximization problem. Optim. Lett. doi:10.1007/s11590-015-0981-5 